\title{
Sight-threatening pseudotumour cerebri associated with excess vitamin A supplementation
}

\author{
James D Benzimra, ${ }^{1}$ Sumu Simon, ${ }^{1}$ Alexandra J Sinclair, ${ }^{2}$ \\ Susan P Mollan ${ }^{1,2}$
}

${ }^{1}$ Birmingham NeuroOphthalmology Unit, Ophthalmology Department, University Hospitals Birmingham NHS Trust, Queen Elizabeth Hospital Birmingham, Birmingham, UK ${ }^{2}$ Department of Neurotrauma and Neurodegeneration, School of Clinical and Experimental Medicine, College of Medical and Dental Sciences, The Medical School, The University of Birmingham, Birmingham, UK

\section{Correspondence to} Susan Mollan, Birmingham Neuro-Ophthalmology Unit, Ophthalmology Department, University Hospitals Birmingham NHS Trust, Queen Elizabeth Hospital Birmingham, Mindelsohn Way, Edgbaston, Birmingham B15 2WB, UK; soozmollan@doctors.org.uk

Accepted 3 August 2014 Published Online First 21 August 2014

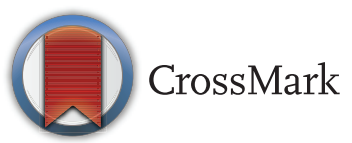

To cite: Benzimra JD, Simon $\mathrm{S}$, Sinclair AJ, et al. Pract Neurol 2015;15: 72-73.

\section{CASE}

A 17-year-old girl presented with a 2 -week history of increasingly severe generalised headache that was worse on waking, with blurring of vision and transient visual obscurations. Her visual acuity was $6 / 24$ right eye and 6/36 left eye with a marked constriction of visual fields. Fundus examination showed severe optic disc swelling bilaterally with a partial macular star appearance (figure 1A, B). Ophthalmic examination revealed no cause for optic disc swelling and neurological examination was otherwise normal. MRI and venography showed no space-occupying lesion, venous thrombosis or stenosis. Full blood count and renal function were normal. Cerebrospinal fluid opening pressure was $77 \mathrm{~cm}$ of CSF (lateral decubitus position) with normal constituents. Body mass index was $24 \mathrm{~kg} / \mathrm{m}^{2}$ (ideal range $18.5-$ $24.9 \mathrm{~kg} / \mathrm{m}^{2}$ ) and her initial history did not identify any causes of elevated intracranial pressure. Based on these findings we diagnosed her with pseudotumour cerebri.

She underwent an urgent lumboperitoneal shunt to prevent further visual loss. At 2-month follow-up she was symptomatically better; visual acuity had improved to $6 / 9$ bilaterally and the papilloedema had resolved (figure $1 \mathrm{G}, \mathrm{H}$ ). At this review, on direct questioning, she admitted to using several dietary supplements for 2 months before initial presentation. These included a high dose multivitamin containing vitamin A $2400 \mu \mathrm{g}$ (8000 IU), $300 \%$ of the recommended daily allowance and others with unspecified vitamin A content. She had stopped these on admission to hospital.

\section{DISCUSSION}

Pseudotumour cerebri may be idiopathic or secondary. Clinicians must take care to exclude secondary causes of raised intracranial pressure in all patients, but in particular in men, children and women of normal body mass index. This patient was not obese and did not fit the typical phenotype for idiopathic intracranial hypertension; her case highlights the importance of specifically asking about dietary intake and supplements when evaluating a patient with pseudotumour cerebri.

There is a well-documented association between hypervitaminosis A and pseudotumour cerebri: either through supplementation of vitamin $\mathrm{A}$, excessive ingestion of liver rich in vitamin $\mathrm{A}$ or synthetic vitamin A derivatives. ${ }^{1}$ In this case, the only identified association was excess dietary supplement vitamin A intake. There is debate about the level at which acute toxicity can occur; some have reported levels below the recommended daily allowance of vitamin A, and measurement of serum retinol does not correlate with vitamin A status during toxicity. $^{2}$

In this case, there were hard exudates in the macula between the optic disc and the fovea in a partial star configuration (figure 1A, B). A macular star most frequently occurs in the setting of idiopathic or infective neuroretinitis; but can occur in any case of optic disc swelling, reflecting the severity. Visual acuity measurements in isolation can be misleading in monitoring these cases, as both the macular star and fluid tracking from the optic disc to the macula can degrade the vision, in addition to the optic neuropathy resulting from severe papilloedema. Here, optical coherence tomography helped in monitoring both the disc and the macula and showed the resolution of papilloedema (figure $1 \mathrm{G}, \mathrm{H}$ ).

Contributors JDB, AJS, SS and SPM were involved in the clinical care of this case. JDB prepared the 


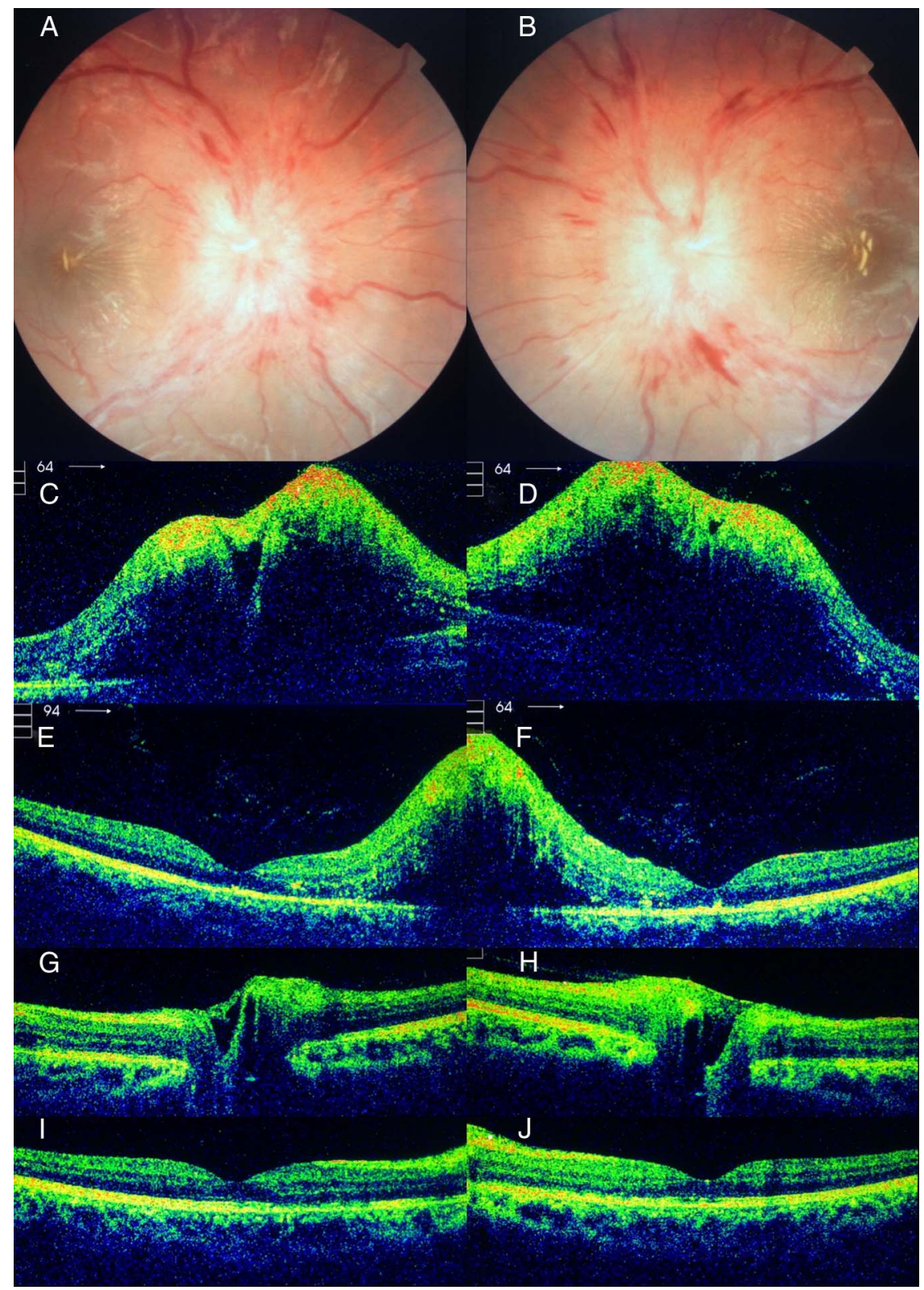

Figure 1 (A and B) Colour fundus photographs showing severe papilloedema with yellow macular exudates in a partial star configuration. ( $C$ and D) Optic disc optical coherence tomograms at presentation show the extent of optic disc oedema. (E and F) Macular optical coherence tomograms at presentation show changes in macular anatomy. $(G$ and $H)$ Optic disc optical coherence tomograms 2 months after lumboperitoneal shunting show resolution of optic disc oedema. (I and J) Macular optical coherence tomograms 2 months after lumboperitoneal shunting show improvement in macular anatomy.

initial draft manuscript. The manuscript was circulated repeatedly and critical revisions made by AJS, SS and SPM.

\section{Competing interests None.}

Patient consent Obtained.

Provenance and peer review Not commissioned; externally peer reviewed. This paper was reviewed by Benjamin Wakerley, Gloucester, UK.

\section{REFERENCES}

1 Libien J, Blaner WS. Retinol and retinol-binding protein in cerebrospinal fluid: can vitamin A take the "idiopathic" out of idiopathic intracranial hypertension? J Neuroophthalmol 2007;27:253-7.

2 Penniston KL, Tanumihardjo SA. The acute and chronic toxic effects of vitamin A. Am J Clin Nutr 2006;83:191-201. 